\title{
The human body exposed to a magnetotherapy device magnetic field
}

\author{
D. Poljak ${ }^{1}$, S. Sesnic ${ }^{1}$, D. Cavka ${ }^{1}$, M. Titlic ${ }^{2} \&$ M. Mihalj ${ }^{2}$ \\ ${ }^{1}$ University of Split, Split, Croatia \\ ${ }^{2}$ Clinical Hospital Centre, Split, Croatia
}

\begin{abstract}
This paper deals with human exposure to extremely low frequency (ELF) magnetic fields generated by a magnetotherapy device. The problem is twofold, i.e. it implies the assessment of an external ELF magnetic field and other internal parameters related to the human body response to this field (total current, power density, total power). Of particular importance is the current density induced inside the human body as the basic restriction proposed by the relevant international bodies for non-ionizing radiation, such as ICNIRP. ELF magnetic field levels generated from a magnetotherapy device are obtained from measurements. Knowing the external magnetic field generated by a magnet, the circular current density induced in the human body is obtained by the use of the analytical formula arising from the disk model of the human torso. On computing the circular current density, it is possible to assess other parameters of interest related to body response. The obtained values of both the magnetic field and the internal current density are compared to exposure limits in terms of reference levels and basic restrictions proposed by ICNIRP.

Keywords: magnetotherapy, human exposure to magnetic fields, disk model of the body, induced current density.
\end{abstract}

\section{Introduction}

Magnetotherapy based on extremely low frequency (ELF) magnetic fields is considered to be a non-invasive technique, widely used in the treatment of muscle pain [1]. The sale of magnetic field based devices such as bracelets, insoles, pillows, etc. makes more than 1 billion dollars worldwide per year [1]. 
Human exposure to ELF magnetic fields generated by magnetotherapy devices has been analyzed in a number of studies in last decade [1-5]. Of particular interest was a possible effect of such exposures to the cardiovascular system [1]. Contradictory findings have been reported in many previous papers. Some studies claim that ELF magnetic fields cause slowing of the heart rate, while other studies do not confirm those effects. For example, the results

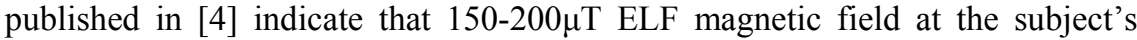
heart position causes slowing of the heart rate. Some authors claim that the slowing of the heart rate due to the exposure to an ELF magnetic field is related to the interaction with the control mechanisms for thermoregulation and blood pressure [2]. Findings in [2] imply the magnetic field $28 \mu \mathrm{T} / 50 \mathrm{~Hz}$ affects the normal heart rate [3]. It is important to emphasize that this value is below the relevant guidelines proposed by ICNIRP for public exposure. There are also experimental studies reporting no significant effects of ELF magnetic fields to which humans are normally exposed daily on heart rate [3].

As the displacement currents at extremely low frequencies are negligible, the electric and magnetic fields can be analyzed separately and can be determined via the computations or/and measurements in [6-10]. In the case of magnetic field exposure, the internal currents form close loops, contrary to electric field exposure where the currents induced in the body have the axial character [7].

This paper deals with an analysis of human exposure to ELF magnetic fields generated by magnetotherapy devices using the analytical approach based on the disk model of the human body [11].

First, the spatial distribution of the magnetic field is determined from measurements and then the circular current density induced in the human body, as a main parameter for the estimation of low frequency exposure effects proposed by ICNIRP basic restrictions [12], is computed. This current density is evaluated by using analytical formula featuring the disk model of the human body. The obtained values for both external magnetic induction and internal current density are compared to exposure limits proposed by ICNIRP.

The knowledge of the internal current density provides further analytical evaluation of the induced total current, power density and the total power dissipated in the human body exposed to ELF magnetic field. It is worth noting that the main feature of the model is efficiency and rather rapid estimation of the phenomena.

\section{The disk model of the human body}

If human being is exposed to ELF magnetic field the circular current density is induced inside the body due to component of the magnetic flux density normal to the body. This internal current density can be assessed by using the simplified disk model of the human body [11], Fig 1.

The disk is assumed to be homogeneous with radius $a$ and conductivity $\sigma$. The analytical relation for the current density can be directly derived from Maxwell equation: 


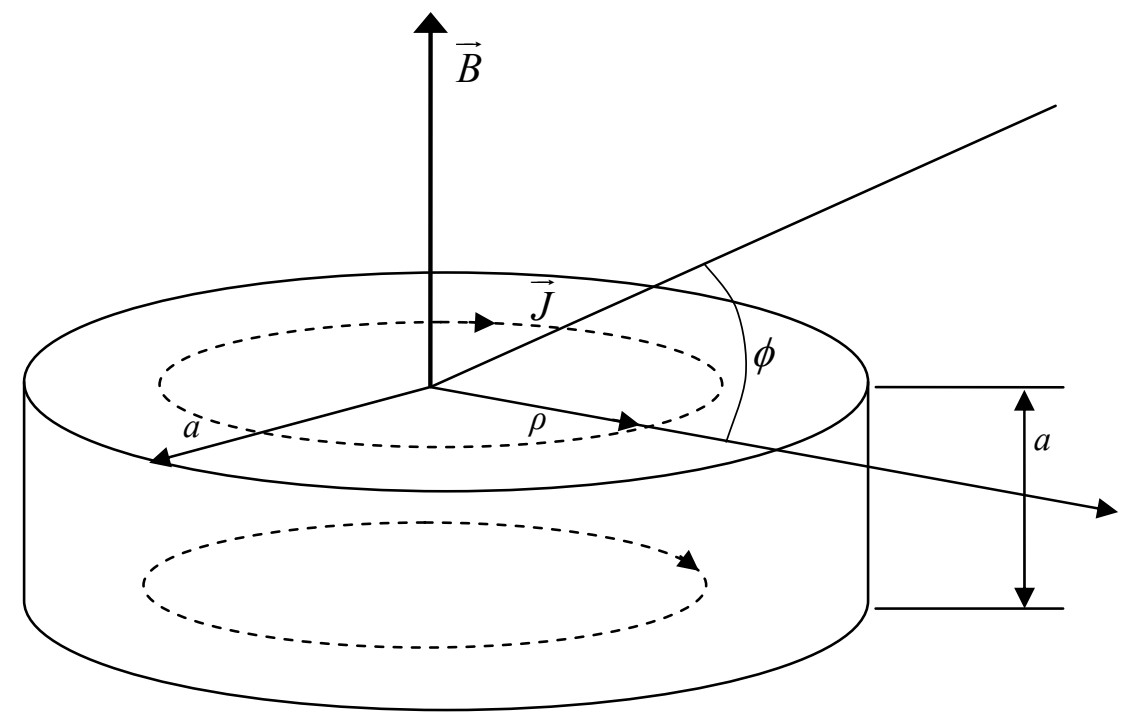

Figure 1: The disk representation of the human body.

$$
\nabla x \vec{E}=-\frac{\partial \vec{B}}{\partial t}
$$

where $\vec{B}$ and $\vec{E}$ denotes the external magnetic and internal electric field, respectively.

Using the constitutive equation:

$$
\vec{J}=\sigma \vec{E}
$$

where $\vec{J}$ denotes the internal current density, and integrating over an arbitrary surface it follows:

$$
\int_{S} \nabla x \vec{J} d \vec{S}=-\int_{S} \sigma \frac{\partial \vec{B}}{\partial t} d \vec{S}
$$

Applying the Stokes theorem, for time harmonic fields assuming the constant value of magnetic induction, equation (3) becomes:

$$
\oint_{c} \vec{J} d \vec{s}=-j \omega \sigma \vec{B} \int_{S} d \vec{S}
$$

i.e., it follows:

$$
\int_{0}^{2 \pi} J_{\phi} \rho d \phi=-j \omega \sigma B_{z} \int_{0}^{\rho} \int_{0}^{2 \pi} \rho d \rho d \phi
$$

where $\omega=2 \pi f$ is the angular operating frequency.

Taking into account the rotational symmetry of the geometry from Fig 1 the straightforward integration yields: 


$$
J_{\phi} \cdot 2 \pi \rho=-j \omega \sigma B_{z} \rho^{2} \pi
$$

and the value of the induced current inside the disk is simply given by:

$$
\left|J_{\phi}\right|=\sigma \pi \rho f \cdot B_{z}
$$

Once knowing the current density, taking the straightforward integration it is possible to calculate the total current flowing through the disk, as shown in Fig 2:

$$
I=\int_{S} \vec{J} d \vec{S}=\int_{0}^{a} \int_{0}^{a} \sigma \pi f B_{z} \rho d \rho d z=\sigma \pi f B_{z} \int_{0}^{a} \int_{0}^{a} \rho d \rho d z=\sigma \pi f B_{z} \frac{a^{3}}{2}
$$

It is worth noting that the thickness of the disk is not specified by the ICNIRP guidelines, as the total current is not related to basic restriction. In this work, the thickness is assumed to be equal $a$ (the disk radius).

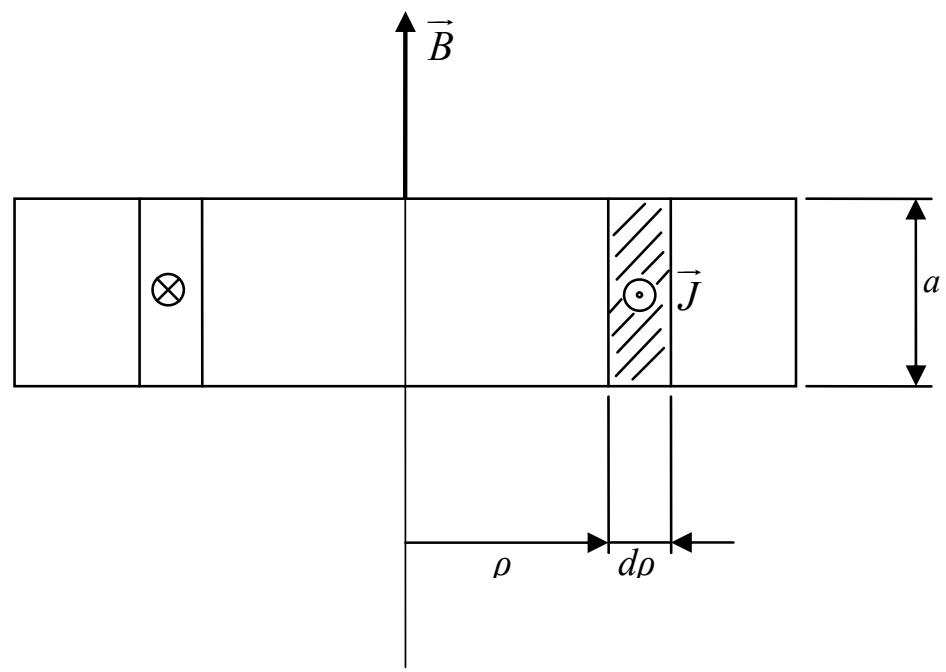

Figure 2: Integration over the disk cross-section.

The power density dissipated inside the body, for time-harmonic excitations, is defined, as follows:

$$
P_{d}=\frac{1}{2} \vec{E} \vec{J}=\frac{|\vec{J}|^{2}}{2 \sigma}
$$

Combining (7) and (9), for $\rho=a$, yields:

$$
P_{d}=\frac{1}{2} \sigma \pi^{2} f^{2} a^{2} B_{z}^{2}
$$


Furthermore, the total power dissipated in the disk body model is given by:

$$
P=\int_{V} P_{d} d V=\int_{V} \frac{1}{2} \vec{E} \vec{J} d V=\int_{V}^{|\vec{J}|^{2}} \frac{}{2 \sigma} d V
$$

Combining (7) and (11) after integration, one obtains:

$$
P=\frac{1}{2 \sigma} \int_{0}^{2 \pi} \int_{0}^{a} \int_{0}^{a} \sigma^{2} \pi^{2} a^{2} B_{z}^{2} \rho d \rho d z d \phi=\frac{1}{4} \sigma \pi^{3} f^{2} B_{z}^{2} a^{5}
$$

Finally, a rate of magnetic energy increase in the human body can be computed from the expression:

$$
\frac{\partial W_{m a g}}{\partial t}=\frac{\partial}{\partial t} \int_{V} \frac{1}{2}(\vec{H} \cdot \vec{B}) d V
$$

As the human body represents a linear magnetic material, the magnetic field strength $\vec{H}$ is proportional to flux density $\vec{B}$ so equation (13) simplifies into:

$$
\frac{\partial W_{m a g}}{\partial t}=\frac{\partial}{\partial t} \int_{V} \frac{B^{2}}{2 \mu} d V
$$

For the time-harmonic variation of external ELF magnetic induction:

$$
B(t)=B_{0} \cos \omega t
$$

It follows:

$$
\left|\frac{\partial W_{m}}{\partial t}\right|=\left|\frac{\partial}{\partial t} \int_{V} \frac{1}{2 \mu} B(t)^{2} d V\right|=\frac{a^{3} \pi}{2 \mu} \omega B_{0}^{2} \sin 2 \omega t
$$

Note that the energy change oscillates twice faster than the external ELF magnetic flux density.

\section{The results}

The measurements of the ELF magnetic field generated by the magnetotherapy device have been carried out via the magnetic field meter AARONIA AG SPECTRAN NF 5020 at the frequencies commonly used for physical therapy, i.e. at $50 \mathrm{~Hz}, 75 \mathrm{~Hz}$ and $100 \mathrm{~Hz}$. The duration of the physical therapy is usually between 15 and 20 minutes. The magnetic flux density measurement has been performed in the vicinity of the device. The obtained values of the magnetic induction are mostly from $100 \mu \mathrm{T}$ to $500 \mu \mathrm{T}$ which directly correspond to ICNIRP reference levels for general and professional population, respectively. Once the magnetic flux density is determined, the internal current density can be calculated using the disk model of the human body. The disk radius is a $=0.14 \mathrm{~m}$ while the conductivity is $\sigma=0.5 \mathrm{~S} / \mathrm{m}$. Furthermore, knowing the internal current density provides the assessment of additional parameters describing the human body response in terms of total current, power density and total power dissipated inside the human body. 
The computed values for various parameters representing the human body response to ELF magnetic field are presented in Tables 1 to 3 for different frequencies. Finally, Figs 3 to 5 show the rate of change of magnetic energy in the disk body model.

Table 1: $\quad$ Various parameters of human body response to ELF magnetic field exposure at $f=50 \mathrm{~Hz}$.

\begin{tabular}{|c|c|c|c|c|c|c|}
\hline $\begin{array}{c}B_{0} \\
{[\mu \mathrm{T}]}\end{array}$ & $\begin{array}{c}\text { ICNIRP } \\
{[\mu \mathrm{T}]} \\
\end{array}$ & $\begin{array}{c}J \\
{\left[\mathrm{~mA} / \mathrm{m}^{2}\right]}\end{array}$ & $\begin{array}{l}\text { ICNIRP } \\
{\left[\mathrm{mA} / \mathrm{m}^{2}\right]}\end{array}$ & $\begin{array}{c}I \\
{[\mathrm{~mA}]} \\
\end{array}$ & $P[\mathrm{nW}]$ & $\begin{array}{c}P_{d} \\
{\left[\mu \mathrm{W} / \mathrm{m}^{3}\right]}\end{array}$ \\
\hline 100 & \multirow{5}{*}{$\begin{array}{c}500 \\
\text { (workers) } \\
100 \\
\text { (general } \\
\text { public) }\end{array}$} & 1,10 & \multirow{5}{*}{$\begin{array}{c}10 \text { (workers) } \\
2 \text { (general public) }\end{array}$} & 0,01 & 5,21 & 1,21 \\
\hline 200 & & 2,20 & & 0,02 & 20,8 & 4,84 \\
\hline 300 & & 3,30 & & 0,03 & 46,9 & 10,9 \\
\hline 400 & & 4,40 & & 0,04 & 83,4 & 19,3 \\
\hline 500 & & 5,50 & & 0,05 & 130,07 & 30,2 \\
\hline
\end{tabular}

Table 2: $\quad$ Various parameters of human body response to ELF magnetic field exposure at $f=75 \mathrm{~Hz}$.

\begin{tabular}{|c|c|c|c|c|c|c|}
\hline $\begin{array}{c}B_{0} \\
{[\mu \mathrm{T}]} \\
\end{array}$ & $\begin{array}{c}\text { ICNIRP } \\
{[\mu \mathrm{T}]} \\
\end{array}$ & $\begin{array}{c}J \\
{\left[\mathrm{~mA} / \mathrm{m}^{2}\right.} \\
\end{array}$ & $\begin{array}{l}\text { ICNIRP } \\
{\left[\mathrm{mA} / \mathrm{m}^{2}\right]} \\
\end{array}$ & $\begin{array}{c}I \\
{[\mathrm{~mA}]} \\
\end{array}$ & $P[\mathrm{nW}]$ & $\begin{array}{c}P_{d} \\
{\left[\mu \mathrm{W} / \mathrm{m}^{3}\right]} \\
\end{array}$ \\
\hline 100 & \multirow{5}{*}{$\begin{array}{c}333 \\
\text { (workers) } \\
67 \\
\text { (general } \\
\text { public) }\end{array}$} & 1,65 & \multirow{5}{*}{$\begin{array}{c}10 \text { (workers) } \\
2 \text { (general public) }\end{array}$} & 0,02 & 11,7 & 2,72 \\
\hline 200 & & 3,30 & & 0,03 & 46,9 & $10,9 \mathrm{E}$ \\
\hline 300 & & 4,95 & & 0,05 & 106 & 24,5 \\
\hline 400 & & 6,60 & & 0,06 & 188 & 43,5 \\
\hline 500 & & 8,25 & & 0,08 & 293 & 68 \\
\hline
\end{tabular}

Table 3: $\quad$ Various parameters of human body response to ELF magnetic field exposure at $f=100 \mathrm{~Hz}$.

\begin{tabular}{|c|c|c|c|c|c|c|}
\hline $\begin{array}{c}B_{0} \\
{[\mu \mathrm{T}]}\end{array}$ & $\begin{array}{c}\text { ICNIRP } \\
{[\mu \mathrm{T}]} \\
\end{array}$ & $\begin{array}{c}J \\
{\left[\mathrm{~mA} / \mathrm{m}^{2}\right]} \\
\end{array}$ & $\begin{array}{l}\text { ICNIRP } \\
{\left[\mathrm{mA} / \mathrm{m}^{2}\right]}\end{array}$ & $\begin{array}{c}I \\
{[\mathrm{~mA}}\end{array}$ & $P[\mathrm{nW}]$ & $\begin{array}{c}P_{d} \\
{\left[\mu \mathrm{W} / \mathrm{m}^{3}\right]}\end{array}$ \\
\hline 100 & \multirow{5}{*}{$\begin{array}{c}250 \\
\text { (workers) } \\
50 \\
\text { (general } \\
\text { public) }\end{array}$} & 1,10 & \multirow{5}{*}{$\begin{array}{c}10 \text { (workers) } \\
2 \text { (general public.) }\end{array}$} & 0,01 & 5,21 & 1,21 \\
\hline 200 & & 4,40 & & 0,04 & 83,4 & 19,3 \\
\hline 300 & & 6,60 & & 0,06 & 188 & 43,5 \\
\hline 400 & & 8,80 & & 0,09 & 334 & 77,4 \\
\hline 500 & & 11,00 & & 0,11 & 521 & 121 \\
\hline
\end{tabular}

From the obtained results it is visible that for some scenarios the values of both magnetic flux density and internal current density exceed the exposure limits, for both general population and workers.

However, this statement should be considered rather carefully, namely:

- According to ICNIRP guidelines the reference levels are assumed to be spatially averaged over the whole body, provided that the basic restrictions on localized exposure are not exceeded. 
- On the other hand, ICNIRP guidelines define public exposure as all kind of exposures to which general population is exposed exceeding occupational exposure and exposure during medical treatment.

- Therefore, an attention should be focused to exposure of workers, i.e. medical personnel.

Scenario of interest is twofold:

- exceeded reference levels for the case of entire body exposure

- exceeded basic restrictions for the case of localized exposure

Consequently, careful future studies on professional exposure to ELF magnetic fields would be of interest.

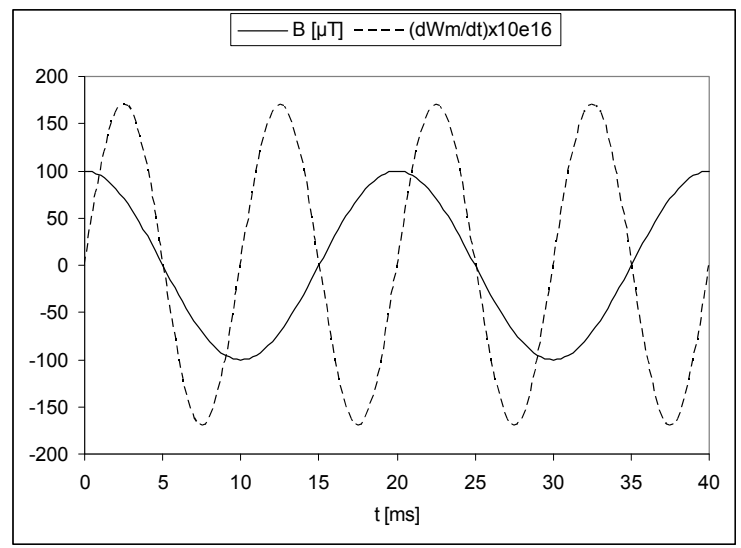

Figure 3: $\quad$ Magnetic induction and magnetic energy change at $f=50 \mathrm{~Hz}$.

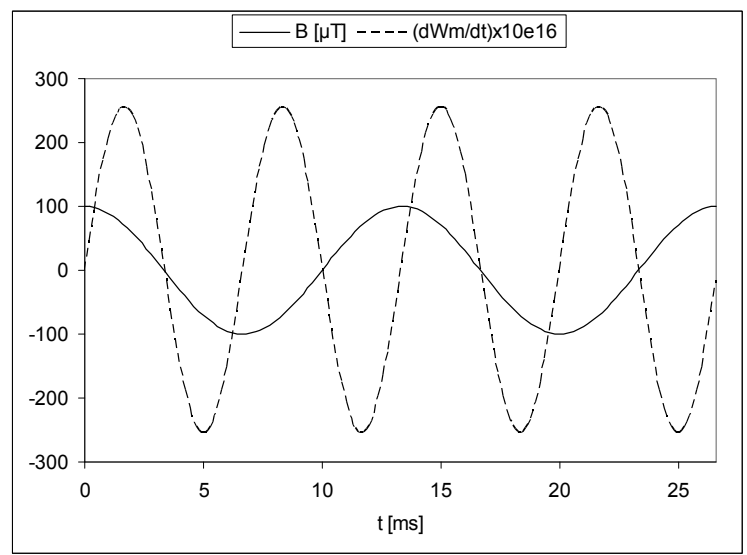

Figure 4: $\quad$ Magnetic induction and magnetic energy change at $f=75 \mathrm{~Hz}$. 


\section{Concluding remarks}

In this work human exposure to ELF magnetic fields generated by magnetotherapy devices is analyzed using the simplified disk model of the human body. The problem is twofold involving the assessment of both magnetic flux density and the internal parameters related to the human body. These parameters are internal current density, power density and total power dissipated in the body. The magnetic field generated by the magnetotherapy device is determined from measurement. The current density induced inside the human body is computed featuring the analytical formula arising from the disk body model. The assessment of the internal current density provides the calculation of further parameters of the human body exposed to ELF magnetic fields in terms of total internal current, power density and total power dissipated inside the body. The results obtained from measurement and calculations for the magnetic flux density and the internal current density, respectively, are below the reference levels and basic restrictions, respectively, proposed by the ICNIRP guidelines in most cases.

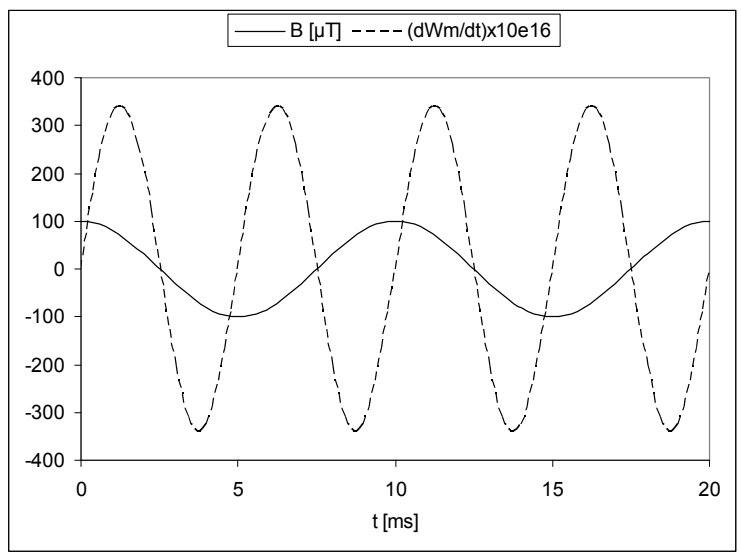

Figure 5: $\quad$ Magnetic induction and magnetic energy change at $f=100 \mathrm{~Hz}$.

Some scenarios may lead to values of external magnetic flux density and internal current density exceeding the exposure limits. Thus, of particular interest would be further investigation on professional exposures to ELF magnetic fields used in physical therapy treatments.

\section{References}

[1] M. Fernandez, P.J. Watson, D.J. Rowbotham, Effect of Pulsed Magnetic Field Therapy on Pain Reported by Human Volunteers in a Laboratory Model of Acute Pain, British Journal of Anaesthesia 99 (2), pp. 266-269, 2007. 
[2] M. L. Sait, A.W. Wood, H.A. Sadafi, A study of Heart Rate Variability in Human Subjects Exposed to Occupational Levels of $50 \mathrm{~Hz}$ Circularly Polarised Magnetic Fields, Medical Engineering and Physics 21, pp. 361369, 1999.

[3] Y. Kurokawa, H. Nitta, H. Imai, M. Kabuto, Can Extremely Low Frequency Alternating Magnetic Fields Modulate Heart Rate or its Variability in Humans?, Autonomic Neuroscience: Basic and Clinical, pp. 53-61, 2003.

[4] Z. Tabor, J. Michalski, E. Rokita, Influence of $50 \mathrm{~Hz}$ Magnetic Field on Human Rate Variability: Linear and Nonlinear Analysis, Bioelectromagnetics 25, pp 474-480, 2004.

[5] L. Finegold, B.L. Flamm, Editorial: Magnet Therapy, Extraordinary claims, but no proved Benefits, BMJ, Vol, 332, Jan 2006.

[6] O.P. Gandhi, J.Y. Chen, Numerical Dosimetry at Power Line Frequencies Using Anatomically Based Models, Bioelectromagnetics Suppl., Vol. 1, pp. 43-60, 1992.

[7] D. Poljak, Human Exposure to Electromagnetic Fields, SouthamptonBoston: WIT Press, 2003.

[8] R.W.P. King, Fields and Currents in the Organs of the Human Body When Exposed to Power Lines and VLF Transmitters, IEEE Trans. Biomedical Eng., Vol. 45, No 4, pp. 520-530, April 1998.

[9] Poljak, D., Rashed, Y., The Boundary Element Modelling of the Human Body exposed to the ELF Electromagnetic Fields, Engineering Analysis with Boundary Elements, 26, pp 871-875, 2002.

[10] D. Poljak, A. Peratta, C.A. Brebbia, A 3D BEM Modelling of Human Exposure to ELF Electric Fields, BEM XVII, Incorporating Electrical Engineering and Electromagnetics, pp. 441-451, Orlando, USA, March 2005.

[11] IEC 62226-2-1: Exposure to electric or magnetic fields in the low and intermediate frequency range - Methods for calculating the current density and internal electric field induced in the human body, 1st edition, November 2004

[12] ICNIRP Guidelines for Limiting Exposure to Time-Varying, Electric, Magnetic and Electromagnetic Fields (up to $300 \mathrm{GHz}$ ), Health Phys., Vol. 74, 4 (1998), 494-522 